\title{
Improved photodecarboxylation properties in zinc photocages constructed using $\boldsymbol{m}$-nitrophenylacetic acid variants
}

\author{
Austin K. Shigemoto, ${ }^{a}$ Avik Bhattacharjee, ${ }^{b}$ Erin E. Hickey, ${ }^{a}$ Hallee Jade Boyd, ${ }^{a}$ Theresa M. \\ McCormick, ${ }^{\mathrm{b}}$ and Shawn C Burdette ${ }^{\mathrm{a} *}$
}

The methoxy- and fluoro-derivatives of meta-nitrophenylacetic acid (mNPA) chromophores undergo photodecarboxylation with comparable quantum yields $(\Phi)$ to unsubstituted $m N P A$, but uncage at red-shifted excitation wavelengths. This observation prompted us to investigate DPAdeCageOMe (2-[bis(pyridin-2ylmethyl)amino]-2-(4-methoxy-3-nitrophenyl)acetic acid) and DPAdeCageF (2-[bis(pyridin-2-ylmethyl)amino]-2-(4-fluoro-3nitrophenyl)acetic acid) as $\mathrm{Zn}^{2+}$ photocages. DPAdeCageOMe has a high $\Phi$ and exhibits other photophysical properties comparable to XDPAdeCage (\{bis[(2-pyridyl)methyl]amino\}(9-oxo-2-xanthenyl) acetic acid), the best perforiming $\mathrm{Zn}^{2+}$ photocage reported to date. Since the synthesis of DPAdeCageOMe is more straightforward than XDPACage, the new photocage will be a highly competitive tool for biological applications.

We are developing decarboxylation reactions of metanitrophenylacetatic acid ( $m$ NPA) and xanthone (XAN) groups to design photocages that release $\mathrm{Zn}^{2+}$ upon irradiation with light. ${ }^{1,2}$ Such photocaged complexes block the biological activity of metal ions until the $\mathrm{Zn}^{2+}$ release is initiated by exposure to light of a specific wavelength. ${ }^{3}$ We recently described DPAdeCage (1) and XDPAdeCage (2), two $\mathrm{Zn}^{2+}$ photocages that incorporate a dipyridyl amine (DPA) chelating group to selectively bind $\mathrm{Zn}^{2+}$ over other common loosely bound metal ions found in cells (Error! Reference source not found.). ${ }^{2}$ Irradiation at or near the $\lambda_{\max }$ initiates a photoreaction that results in the loss of the carboxylic acid functional group as $\mathrm{CO}_{2}$ which shifts the $\mathrm{Zn}^{2+}$ binding equilibrium toward unbound metal ion (Scheme 1). The transformation from a 4-coordinate to a 3coordinate chelator leads to a $10^{5}$-fold reduction in binding affinity $\left(\Delta K_{d}\right)$. For biological applications, a rapid synthesis to produce photocages in high yields would be ideal. The photophysics of XAN chromophores are superior to those of mNPA groups, ${ }^{4-6}$ but the low-yielding synthesis of the XAN scaffolding limits the pace of photocage development. To overcome these limitations, we functionalized several $m$ NPA chromophores to develop DPAdeCage derivatives with improved photophysical properties. In addition to those improvements, the added functional groups facilitate selective

\footnotetext{
Department of Chemistry and Biochemistry, Worcester Polytechnic Institute, 100 Institute Road, Worcester, MA 01609-2280, USA.E-mail: scburdette@wpi.edu.

b. Department of Chemistry, Portland State University, Portland, Oregon, 97201 USA
}

nitration at the 3-position of the aromatic ring simplifying the purification procedure for the mNPA chromophore that will facilitate the rapid generation of photocages.<smiles>O=[N+]([O-])c1cccc(C(CO)N(Cc2ccccn2)Cc2ccccn2)c1</smiles>

$$
\begin{aligned}
\text { DPAdeCage (1) } \\
\lambda_{\max }=261 \mathrm{~nm} \\
\mathrm{~K}_{\mathrm{d}} \mathrm{Zn}^{2+}=0.8 \mathrm{pM} \\
\Delta \mathrm{K}_{\mathrm{d}} \mathrm{Zn}^{2+}=2.0 \times 10^{5}
\end{aligned}
$$$$
\Phi\left(\mathrm{apo} / \mathrm{Zn}^{2+}\right)=40.0 / 5.0
$$<smiles>O=C(O)C(c1ccc2oc3ccccc3c(=O)c2c1)N(Cc1ccccc1)Cc1ccccn1</smiles>

$$
\begin{gathered}
\text { XDPAdeCage (2) } \\
\lambda_{\max }=347 \mathrm{~nm} \\
\mathrm{~K}_{\mathrm{d}} \mathrm{Zn}^{2+}=4.6 \mathrm{pM} \\
\Delta \mathrm{K}_{\mathrm{d}} \mathrm{Zn}^{2+}=3.3 \times 10^{4} \\
\Phi\left(\mathrm{apo} / \mathrm{Zn}^{2+}\right)=20.0 / 27.0
\end{gathered}
$$

Fig 1 Structures and important photophysical properties of the $m$ NPA-based DPAdeCage (1) and XAN-based XDPAdeCage (2), which are both effective $\mathrm{Zn}^{2+}$ photocages. The use of the XAN chromophore red-shifts the uncaging wavelength photocage(Cage) where X stand for xanthone in XDPAdeCage.

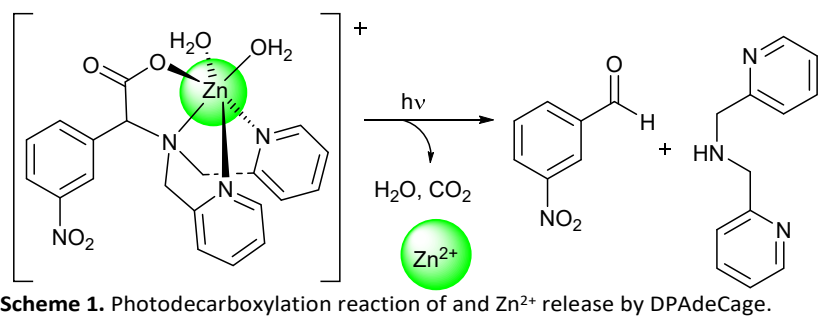

Designing effective photocages requires avoiding short wavelength excitation $\left(\lambda_{\max }\right)$, which can induce cellular photodamage, while still delivering sufficient energy to break chemical bonds. Compared to our mNPA-based DPAdeCage, the $\lambda_{\max }$ of XAN-derived XDPAdeCage is red-shifted by over $80 \mathrm{~nm}$ (Error! Reference source not found.). Furthermore, upon coordination to $\mathrm{Zn}^{2+}$ the photoreactivity of DPAdeCage is significantly reduced. The XAN chromophore offers significant photophysical advantages that include maintaining a high $\Phi$ upon $\mathrm{Zn}^{2+}$ binding. Accessing the XAN chromophore necessary to make XDPAdeCage requires a 2-step synthesis with a maximum $20 \%$ yield. By comparison, the nitration step to synthesize the mNPA chromophore has a much higher yield, increasing the total material obtained ( $70 \%$ yield of $m \mathrm{NPA}$ ). The difference in reaction time, $24 \mathrm{~h}$ and $1 \mathrm{~h}$ respectively, also impacts the rate of photocage synthesis. We hypothesize that 
the relative ease of making $m$ NPA derivatives compared to the XAN chromophore, as well as the numerous readily available starting materials, will facilitate the rapid production of $m$ NPAbased photocages for numerous applications. Furthermore, if functionalization of the mNPA group could shift the $\lambda_{\text {abs }}$ sufficiently, we can prepare and screen $\mathrm{Zn}^{2+}$ photocages for biological applications more quickly as well as expand the toolbox of tunable chromophores.

Table 1. Summary of photophysical data of $m$ NPA derivatives.<smiles>[R]c1ccc(CC(=O)O)cc1[N+](=O)[O-]</smiles>

\begin{tabular}{cccccc}
\multicolumn{1}{c}{$\mathrm{R}$} & $\sigma_{\mathrm{p}}{ }^{\mathrm{a}}$ & $\lambda_{\max }(\mathrm{nm})$ & $\varepsilon\left(\mathrm{M}^{-1}, \mathrm{~cm}^{-1}\right)$ & $\Phi_{\text {Photolysis }}$ \\
\hline 3a & $\mathrm{OAC}$ & 0.31 & 317 & 1478 & 16.3 \\
\hline 3b & $\mathrm{Cl}$ & 0.23 & 315 & 1455 & 8.53 \\
\hline 3c & $\mathrm{F}$ & 0.06 & 311 & 1758 & 24.0 \\
\hline 3d & $\mathrm{HNAc}$ & 0 & 332 & 2123 & 10.2 \\
\hline 3e & $\mathrm{H}$ & 0 & 272 & $7087^{\mathrm{b}}$ & 20.1 \\
\hline 3f & $\mathrm{OMe}$ & -0.27 & 347 & 2589 & 22.6 \\
\hline 3g & $\mathrm{OH}$ & -0.37 & 427 & 2989 & 0.0 \\
\hline 3h & $\mathrm{NH}_{2}$ & -0.66 & 425 & 3729 & 0.0 \\
\hline 3i & $\mathrm{NMe}_{2}$ & -0.83 & 460 & 5915 & 0.0
\end{tabular}

Measurements obtained in $40 \mathrm{mM}$ HEPES buffer $(\mathrm{pH} 7.5,100 \mathrm{mM} \mathrm{KCl})$. Quantum yields were obtained in solutions containing $30 \%$ methanol to maintain

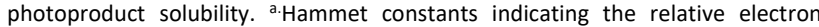
withdrawing or donating properties of each $\mathrm{R}$ group. ${ }^{\mathrm{b}}$.3e lacks a red-shifted absorbance band; $\varepsilon=3264 \mathrm{M}^{-1} \mathrm{~cm}^{-1}$ at $\lambda_{300}, 356 \mathrm{M}^{-1} \mathrm{~cm}^{-1}$ at $\lambda_{350}$.

In previous studies on ortho-nitrobenzyl photocages (oNB), introducing electron donating groups (EDGs) or electron withdrawing groups (EWG) produced photocages with different rates of photolysis, quantum yields and excitation wavelengths. $^{7-11}$ Tsien, Kaplan, and others reported functionalization of the oNB ring with methoxy groups shifted the $\lambda_{\max }$ to longer wavelengths, ${ }^{7,8}$ suggesting we might benefit from a similar approach. Modification of the nitrobenzyl ring, however, can also impact the electronics of the aromatic ring and therefore the nature of the photoreaction. ${ }^{10,12}$ Unlike oNBs that undergo a Norrish type II photoreaction, ${ }^{13,}{ }^{14}$ the mechanism for photodecarboxylation of $m$ NPA chromophores involves an electron transfer from the nitro group to the methylene bridge of the phenylacetate to initiate the photodecarboxylation. ${ }^{15-17}$ This suggests increasing the electron-density around the aromatic ring would impede electron-transfer to the methylene bridge, which could reduce photolysis quantum yields $(\Phi)$. Thus, we predicted EDGs would exhibit reduced photoactivity compared to mNPAs functionalized with EWGs. By adjusting the electronic-structure of the $m$ NPA chromophore we hoped to access a DPAdeCagederivative that will maintain a high $\Phi$ upon coordination to $\mathrm{Zn}^{2+}$.

A series of $m$ NPA chromophores were prepared with various EDGs and EWGs at the 4-position of the aromatic ring (Error! Reference source not found.). We hypothesized that functional groups with heteroatoms would extend the conjugated $\pi$ system and red-shift the $\lambda_{\max }$. Functionalization resulted in the appearance of a new red-shifted absorbance feature that was not observed in the parent $m$ NPA $3 \mathbf{e}$ (Figure 2). The red-shifted feature appears as a shoulder of the strong UV band centred at circa $270 \mathrm{~nm}$ in most of the compounds, but the band shifts sufficiently in the methoxy- (3f) and hydroxy-derivatives (3g) to appear as a distinct new absorbance feature. While all the derivatives possess a weaker extinction coefficient $(\varepsilon)$ of the large absorbance band below $300 \mathrm{~nm}$ compared to $\mathbf{3 e}$, the compounds also absorb more strongly at wavelengths greater than $320 \mathrm{~nm}$ (Error! Reference source not found.).

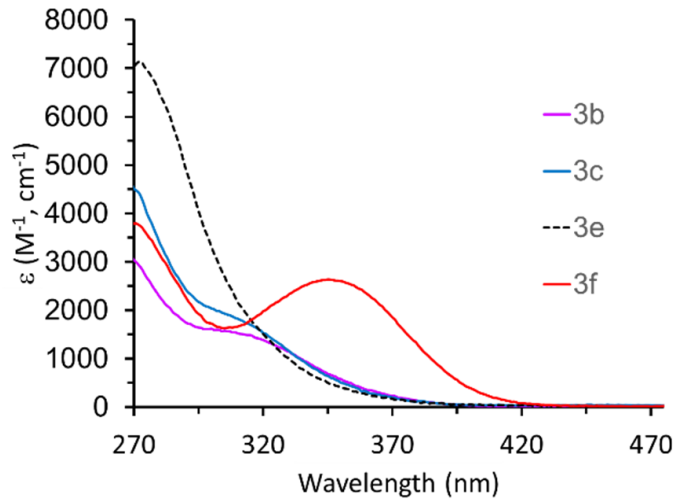

Fig. 2. Absorption spectra of $\mathbf{3 b}(-\mathrm{Cl}), \mathbf{3 c}(-\mathrm{F}), \mathbf{3 e}(-\mathrm{H})$ and $\mathbf{3 f}(-\mathrm{OMe})$ in aqueous solvent and simulated physiological conditions (40 mM HEPES buffer $\mathrm{pH} 7.5100$ $\mathrm{mM} \mathrm{KCl)}$

The photolysis quantum yields $(\Phi)$ were determined under simulated physiological conditions using LC/MS to monitor the loss of 3a-3i. Despite the red-shifted band, the photoactivity of the acetyl (3a), chloro (3b), and amide-functionalized (3d) compounds is diminished relative to $3 e$. The only EWGcontaining derivative that exhibits a $\Phi$ similar to $3 e$ is the fluoroderivative 3c. Although $\mathbf{3 b}$ has a similar chloro group, the observed photoreactivity is diminished relative to 3c. Notably the absorptivity of $\mathbf{3} \mathbf{c}$ is stronger than $\mathbf{3} \mathbf{b}$ across the entire spectrum and likely contributes to the observed difference in $\Phi$ (Error! Reference source not found.). While $\mathbf{3 c}$ exhibits photoactivity comparable to XDPAdeCage, the $\lambda_{\max }$ is blueshifted by over $30 \mathrm{~nm}$.

The mass peaks observed in the LC/MS spectrum of 3a-f after photolysis indicate tolyl and aldehyde derivatives analogous to those previously observed with mNPA-based photocages predominate the photoproducts (Scheme 1). ${ }^{1,2}$ Compounds containing EDGs (3f-3i) exhibit a bathochromic shift in $\lambda_{\max }$ compared to the parent compound $\mathbf{3 e}$; however only $\mathbf{3 f}$ retained photodecarboxylation activity. The hydroxy compound $3 g$ exhibits a higher $\varepsilon\left(2989 \mathrm{M}^{-1}, \mathrm{~cm}^{-1}\right)$ and a more red-shifted absorbance $\left(\lambda_{\max }=427 \mathrm{~nm}\right)$ but lacks photoactivity compared to the corresponding methoxy derivative (3f). Excited state proton transfer does not appear to be responsible for the inactivity of $\mathbf{3 g}$ as the compound remains photochemically inert under strongly basic conditions ( $\mathrm{pH}$ 14). Although $\mathbf{3 f}$ contains an electron-donating methoxy group, the photodecarboxylation $\Phi$ and red-shifted $\lambda_{\max }$ is the most comparable to XDPAdeCage. While the methoxy functional group is electron-donating by 
resonance, inductive effects provide the most plausible explanation for the anomalous behaviour in the EDG series.

The model methoxy- and fluoro-compounds $\mathbf{3 f}$ and $\mathbf{3 c}$ exhibited the most comparable photophysical properties to XDPAdeCage, therefore we chose these chromophores to build new DPAdeCage derivatives (Error! Reference source not found.). Our initial attempts to prepare these photocages via our established route were unsuccessful due to oxidative decomposition during the final nitration reaction. Alternatively, starting with $\mathbf{3 f}$ and $\mathbf{3 c}$ provided $\mathbf{4}$ (DPAdeCageOMe) and $\mathbf{5}$ (DPAdeCageF) in $11.6 \%$ (5 steps) and $11.8 \%$ (4 steps) respectively. The only significant difference between the two synthetic pathways is the final ester hydrolysis. DPAdeCageF required acidic conditions at elevated temperature, while the basic conditions used to prepare DPAdeCage successfully provided DPAdeCageOMe. A combination of product decomposition in the harsher deprotection reaction as well as the more difficult isolation of DPAdeCageF from acidic solution, reduced the overall yield of the final product. A comparison of the synthetic pathways required to develop the new $m$ NPA photocages and XDPAdeCage reveals both DPAdeCageOMe and DPAdeCageF can be more rapidly prepared and in greater amounts than XDPAdeCage.

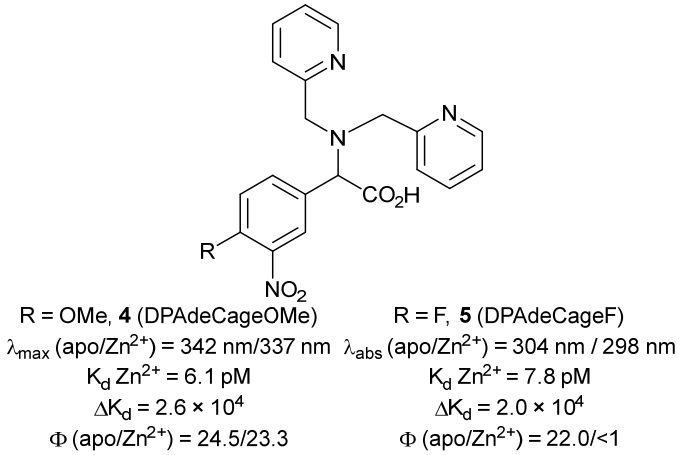

Fig. 3. Structures and important photophysical properties of DPAdeCageOMe (4) and DPAdeCageF (5).

Zinc-binding constants for DPAdeCageOMe and DPAdeCageF were determined by competitive titration with a high affinity zinc ligand compound 4-(p-pyridylazo)resorcinol (PAR). Under simulated physiological conditions (40 mM HEPES, $100 \mathrm{mM} \mathrm{KCl}, \mathrm{pH} 7.5)$, DPAdeCageOMe $\left(K_{d} 6.1 \mathrm{pM}, \Delta \mathrm{K}_{\mathrm{d}} 2.6 \times 10^{4}\right)$ and DPAdeCageF $\left(\mathrm{K}_{\mathrm{d}} 7.8 \mathrm{pM}, \Delta \mathrm{K}_{\mathrm{d}} 2.0 \times 10^{4}\right)$ bind $\mathrm{Zn}^{2+}$ with similar affinity to DPAdeCage ( $\left.\mathrm{K}_{d} 0.8 \mathrm{pM}\right)$. The slight difference in $K_{d}$ has a minimal impact on the $\Delta K_{d}$ but both compounds should efficiently release $\mathrm{Zn}^{2+}$ following irradiation due to changes in the binding equilibrium. The minor difference observed in the $K_{d}$ values is likely due to small inductive effects, caused by the addition of functional groups on the mNPA ring.
Table 1: Photophysical data for apo- and $\mathrm{Zn}^{2+}$-complexes of DPAdeCage, XDPAdeCage, DPAdeCageOMe and DPAdeCageF

\begin{tabular}{cccc} 
& $\lambda_{\max }(\mathrm{nm})$ & $\varepsilon\left(\mathrm{M}^{-1}, \mathrm{~cm}^{-1}\right)$ & $\Phi_{\text {Photolysis }}$ \\
\hline DPAdeCage & 261 & 5120 & 40.0 \\
\hline${\text { [Zn(DPAdeCage) }]^{+}}$ & 262 & 5230 & 5.0 \\
\hline XDPAdeCage & 347 & 4730 & 20.0 \\
\hline${\text { [Zn(XDPAdeCage) }]^{+}}$ & 347 & 5270 & 27.0 \\
\hline DPAdeCageOMe & $262 / 342$ & $9680 / 1686$ & 24.5 \\
${\text { [Zn(DPAdeCageOMe) }]^{+}}^{\text {DPPAdeCageF }}$ & $262 / 337$ & $6670 / 1737$ & 23.3 \\
${\text { [Zn(DPAdeCageF) }]^{+}}^{2}$ & $261 / 304$ & $10871 / 1804$ & 22.0 \\
\end{tabular}

Measurements obtained in $40 \mathrm{mM}$ HEPES buffer $(\mathrm{pH} 7.5,100 \mathrm{mM} \mathrm{KCl})$. Quantum yields were obtained in solutions containing $30 \%$ methanol to maintain photoproduct solubility.

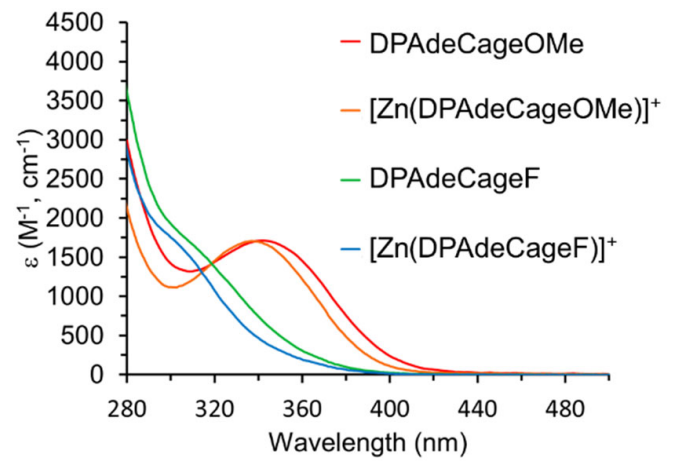

Fig. 4. Absorbance spectrum of DPAdeCageOMe/[Zn(DPAdeCageOMe) $]^{+}$and conditions (40 mM HEPES buffer DPAdeCageOMe has a well-defined, red-shifted absorbance band at $347 \mathrm{~nm}$ that is comparable to XDPAdeCage.

The photophysical properties of DPAdeCageOMe and DPAdeCageF are nearly identical to the parent compounds $\mathbf{3 f}$ and $3 \mathrm{c}$ under aqueous conditions (Error! Reference source not found.). Notably, the $\lambda_{\max }$ of both DPAdeCageOMe and DPAdeCageF are red-shifted relative to DPAdeCage. The methoxy-functionalized DPAdeCageOMe $\left(342 \mathrm{~nm}\right.$ ) has a $\lambda_{\max }$ nearly identical to XDPAdeCage $(347 \mathrm{~nm})$. The addition of $\mathrm{Zn}^{2+}$ to the photocages results in a slight hypsochromic shift of $\lambda_{\max }$, but relatively little change in absorptivity (Table 1). An analysis of the LC/MS spectrum of DPAdeCageOMe and DPAdeCageF following irradiation, indicate successful photodecarboxylation following irradiation. In the absence of $\mathrm{Zn}^{2+}$, both compounds appear to successfully release the carboxylate group based on the change in mass. In DPAdeCage, ${ }^{2}$ a decrease in $\Phi$ is observed upon coordination to $\mathrm{Zn}^{2+}$ and DPAdeCageF exhibits an even more dramatic decrease upon $\mathrm{Zn}^{2+}$ coordination. Only extended irradiation times $(>30 \mathrm{~min})$ of [ $\mathrm{Zn}(\mathrm{DPAdeCageF})]^{+}$resulted in evidence of photolysis, which severely limits the potential applications of the photocage. The addition of $\mathrm{Zn}^{2+}$ does not appear to affect the resulting photoreaction of DPAdeCageOMe; however, DPAdeCageF forms a wider slate of photoproducts in the presence of $\mathrm{Zn}^{2+}$ after long irradiation. Some higher mass, emissive photoproducts suggest that $\mathrm{Zn}^{2+}$ facilitates the formation of coupled DPAdeCageF products 
TD-DFT calculations were performed to probe if the electronic structure contributed to the observed difference in photoreaction of $[\mathrm{Zn}(\mathrm{DPAdeCageOMe})]^{+}$and $[\mathrm{Zn} \text { (DPAdeCageF)] }]^{+}$The structure of DPAdeCageOMe, DPAdeCageF, [Zn(DPAdeCageOMe) $]^{+}$and [Zn(DPAdeCageF) $]^{+}$ were optimized using DFT and the frontier molecular orbitals contributing to the electronic transitions found in the TD-DFT calculations were visualized. In all cases, the lowest energy excitation involves excitation from the HOMO to the LUMO. The LUMOs are localized around the nitro group and phenyl ring for all compounds, consistent with previous studies of $m \mathrm{NPA}$ chromophores (Error! Reference source not found.). ${ }^{17} \mathrm{We}$ believe that promoting an electron into a LUMO localized on the nitro group is necessary for photolysis to occur, suggesting

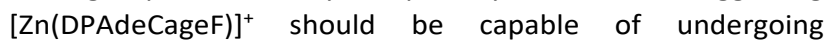
photodecarboxylation based on the calculated electronic structure. A closer examination of the observed oscillator strengths (f) representing the molar absorptivity of $\mathrm{Zn}(\text { DPAdeCageOMe })^{+}(f=0.04)$ and $\mathrm{Zn}(\text { DPAdeCageF })^{+}(f=0.01)$ reveal a nearly 4 -fold decrease in the oscillator strength of the lowest energy transition for Zn(DPAdeCageF) ${ }^{+}$, which may explain a decrease in observed photolysis, although other factors probably contribute to the difference given the large drop in $\Phi$ observed experimentally.

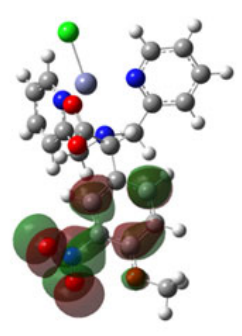

[Zn(DPAdeCageOMe)]

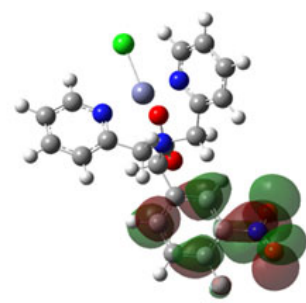

[Zn(DPAdeCageF) $]^{+}$ Fig. 5. Optimized geometry depicting the LUMO of [Zn(DPAdeCageOMe) $]^{+}$and
[Zn (DPAdeCageF) $]^{+}$determined by DFT calculations (B3LYP/6-311+G(d)).

Through a study of model mNPA chromophores, we were able to identify derivatives with comparable photophysical properties compared to the decarboxylation reaction observed with XAN groups. Specifically, fluoro- and methoxy- groups introduce a distint red-shifted absorption band; however, only the methoxy derivative retains sufficient photodecarboxylation activity when integrated into a $\mathrm{Zn}^{2+}$ photocage. We were pleased to see DPAdeCageOMe maintains a high $\Phi$ when coordinated to $\mathrm{Zn}^{2+}$ and has nearly identical photocaging properties to XDPAdeCage. A methoxy group in the 4-position also provides a potential site for modification of future photocages through an ether linkage that should not impact $\mathrm{Zn}^{2+}$ binding significantly and will be the subject of future investigations.

\section{Conflicts of interest}

There are no conflicts to declare.

\section{Acknowledgements}

This work was supported by the National Science Foundation (grant CHE- 2004088) and the Worcester Polytechnic Institute.

\section{Notes and references}

1. P. N. Basa, S. Antala, R. E. Dempski and S. C. Burdette, Angew. Chem., 2015, 127, 13219.

2. P. N. Basa, C. A. Barr, K. M. Oakley, X. Liang and S. C. Burdette, J. Am. Chem. Soc., 2019, 141, 12100.

3. C. Gwizdala and S. C. Burdette, in Inorganic Chemical Biology, ed. G. Gasser, John Wiley \& Sons, New Jersey, 2014, pp. 275.

4. J. A. Blake, E. Gagnon, M. Lukeman and J. Scaiano, Org. Lett., 2006, 8, 1057.

5. J. A. Blake, M. Lukeman and J. C. Scaiano, J. Am. Chem. Soc., 2009, 131, 4127.

6. J. D. Margerum and C. T. Petrusis, J. Am. Chem. Soc., 1969, 91, 2467.

7. G. Ellis-Davies and J. Kaplan, J. Org. Chem., 1988, 53, 1966.

8. S. Adams, J. Kao and R. Tsien, J. Am. Chem. Soc., 1989, 111, 7957.

9. C. G. Bochet, Tet. Lett., 2000, 41, 6341.

10. D. P. Kennedy, D. C. Brown and S. C. Burdette, Org. Lett., 2010, 12, 4486

11. H. K. Agarwal, R. Janicek, S.-H. Chi, J. W. Perry, E. Niggli and G. C. R. Ellis-Davies, J. Am. Chem. Soc., 2016, 138, 3687.

12. H. D. Bandara, T. P. Walsh and S. C. Burdette, Chem. Eur. J., 2011, 17, 3932.

13. A. Blanc and C. G. Bochet, J. Am. Chem. Soc., 2004, 126, 7174.

14. J.-M. Mewes, E. Pepler, J. Wachtveitl and A. Dreuw, J. Phys. Chem. A, 2012, 116, 11846.

15. D. Budac and P. Wan, J. Photochem. Photobiol. A, 1992, 67, 135.

16. P. Wan and S. Muralidharan, J. Am. Chem. Soc., 1988, 110, 4336.

17. P. Wan and K. Yates, Can. J. Chem., 1986, 64, 2076. 